\title{
Uniportal video-assisted thoracoscopic surgery (VATS) for the treatment of early-stage lung cancer with whole pleural adhesion
}

\author{
Si Young Choi^, Youngkyu Moon^ \\ Department of Thoracic \& Cardiovascular Surgery, Eunpyeong St. Mary's Hospital, College of Medicine, The Catholic University of Korea, Seoul, \\ Republic of Korea \\ Contributions: (I) Conception and design: Y Moon; (II) Administrative support: SY Choi; (III) Provision of study materials or patients: Both authors; \\ (IV) Collection and assembly of data: Both authors; (V) Data analysis and interpretation: Y Moon; (VI) Manuscript writing: Both authors; (VII) Final \\ approval of manuscript: Both authors. \\ Correspondence to: Youngkyu Moon, MD, PhD. Department of Thoracic \& Cardiovascular Surgery. Eunpyeong St. Mary's Hospital, College of \\ Medicine, The Catholic University of Korea, 1021, Tongil-ro, Eunpyeong-gu, Seoul, 03312, Republic of Korea. Email: mykae@catholic.ac.kr.
}

Background: Uniportal video-assisted thoracoscopic surgery (VATS) is considered a challenging procedure in cases of lung cancer where the entire pleura and lung are attached (whole pleural adhesion). The purpose of this study was to evaluate the surgical results of uniportal VATS for the treatment of lung cancer with whole pleural adhesion.

Methods: For all patients who underwent VATS lung cancer surgery at a single hospital in Korea, multiportal VATS was performed before 2017 and uniportal VATS was performed after January 2017 by the same surgeons. The surgical results of multiportal VATS and uniportal VATS for the treatment of lung cancer with whole pleural adhesion were compared retrospectively.

Results: There were 16 eligible uniportal VATS cases and 57 multiportal VATS cases. There were no significant differences of clinicopathologic characteristics between the two groups. There were 5 conversions to open thoracotomy in the multiportal VATS group and no conversion to open thoracotomy in the uniportal group $(\mathrm{P}=0.579)$. The perioperative and postoperative outcomes were not different between the two groups. There was no mortality in all patients. For patients undergoing VATS lobectomy, the perioperative and postoperative outcomes were similar after uniportal and multiportal VATS lobectomy. For all patients, the 3-year recurrence-free survival (RFS) was $80.0 \%$ after uniportal VATS and $79.5 \%$ after multiportal VATS $(\mathrm{P}=0.951)$ and for patients with stage I non-small cell lung cancer (NSCLC), the 3-year RFS after uniportal VATS was $91.7 \%$ and the 3-year RFS after multiportal VATS was 89.3\% ( $\mathrm{P}=0.999)$. Uniportal VATS was not a significant risk factor for recurrence in the multivariate analysis.

Conclusions: Uniportal VATS was not inferior to multiportal VATS in surgical outcome or shortterm prognosis in resection of lung cancer with whole pleural adhesion. Whole pleural adhesion is not a contraindication to uniportal VATS, which has been safe and feasible in cases of lung cancer with whole pleural adhesion.

Keywords: Uniportal video-assisted thoracoscopic surgery (VATS); lung cancer; pleural adhesion

Submitted Aug 08, 2021. Accepted for publication Dec 02, 2021.

doi: $10.21037 /$ tcr-21-2113

View this article at: https://dx.doi.org/10.21037/tcr-21-2113

^ ORCID: Si Young Choi, 0000-0002-7372-6959; Youngkyu Moon, 0000-0002-6875-6918. 


\section{Introduction}

Uniportal video-assisted thoracoscopic surgery (VATS) is the most advanced minimally invasive type of lung surgery (1). It was initially adopted for pulmonary wedge resection, and uniportal VATS lobectomy was first reported by Diego Gonzalez-Rivas $(2,3)$ in 2011. Since that initial report, uniportal VATS has been actively studied and used in anatomical pulmonary resection in the treatment of lung cancer (1,4-6). Uniportal VATS has been considered more difficult than multiportal VATS because it is performed by inserting 2 or 3 surgical instruments and the thoracoscope through a single incision.

By 2017, Gonzalez-Rivas had proposed a standardized technique for uniportal VATS anatomical pulmonary resection $(7,8)$, and a uniportal VATS training program had been established at the Shanghai Pulmonary hospital in Shanghai, China (9), an ultra-high-volume center for thoracic surgery, where more than 10,000 lung cancer resections are performed every year. We are among the many surgeons who have participated in the uniportal VATS training program and who are now conducting uniportal VATS. Since January 2017, when we participated in the training program, we have been performing all lung cancer surgeries with uniportal VATS (10).

In some cases of whole pleural adhesion, where the entire pleura and lung are attached, there may not even be space for insertion of the thoracoscope. Although multiportal VATS lobectomy with pleural adhesiolysis is possible in most cases of operable lung cancer, it can be very time consuming to separate the adhesion of the entire lung from the parietal pleura. Lung cancer surgery with whole pleural adhesion is a challenging procedure for multiportal VATS (11). We believed it would be even more challenging in uniportal VATS, as we surmised that it would be too difficult to separate the entire adhesion from only one direction through a single port. However, our thinking changed after we completed the uniportal VATS training program, and we have now been performing uniportal VATS in all lung cancer patients with whole pleural adhesion since January 2017.

The purpose of this study was to evaluate the surgical results of uniportal VATS in the treatment of lung cancer with whole pleural adhesion and to examine whether uniportal VATS can be regarded as a feasible surgical approach for lung cancer with whole pleural adhesion. We hypothesized that uniportal VATS did not have worse surgical or oncological outcomes than multiportal VATS.
We present the following in accordance with the STROBE reporting checklist (available at https://tcr.amegroups.com/ article/view/10.21037/tcr-21-2113/rc).

\section{Methods}

\section{Patients}

From 2012 to 2018 at a single institution, 727 patients were diagnosed with non-small cell lung cancer (NSCLC) and underwent curative surgery by two surgeons including the author at a hospital in Korea. We performed multiportal VATS from 2012 to 2016 and uniportal VATS from 2017 to 2018. There were 100 patients with whole pleural adhesion. Among them, 27 were excluded from the retrospective analysis because of open thoracotomy (3 cases), incomplete resection ( 2 cases), higher than stage III (10 cases), or preoperative chemotherapy (12 cases). Whole pleural adhesion is defined as the complete $(100 \%)$ attachment of the entire parietal and visceral pleura. To reduce the selection bias, all data were obtained from consecutive patients. Finally, 73 consecutive patients were included.

The operations were conducted by two surgeons who had sufficient experience with multiportal VATS. They began performing uniportal VATS after attending the uniportal VATS training program at the Shanghai Pulmonary Hospital in January 2017, and they have operated all indicated lung cancers since 2017 with uniportal VATS. In this study, the surgical results of multiportal VATS before January 2017 and those of uniportal VATS after January 2017 were compared. The patients were divided into a uniportal VATS group and a multiportal VATS group. The clinicopathologic characteristics were analyzed in the two groups and perioperative and postoperative outcomes were compared.

The study was conducted in accordance with the Declaration of Helsinki (as revised in 2013). It was approved by the institutional review board of Eunpyeong St. Mary's Hospital at the Catholic University of Korea (No. PC21RISI0115) and individual consent was waived.

\section{Surgical procedures}

Most clinical stage I and II lung cancers are operated with VATS in our institution. Operative procedures in the study included wedge resection, segmentectomy, lobectomy, and bilobectomy. Multiportal VATS was performed with 3 ports and a working incision, whereas uniportal 


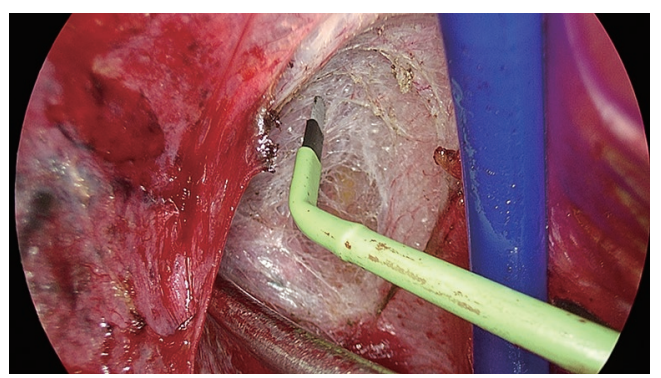

Figure 1 The thoracoscope is inserted into the working incision after blunt dissection to secure a space around the incision.

VATS was performed using only the working incision. In multiportal VATS, the camera and several instruments are placed in separate ports to perform the surgery, whereas in uniportal VATS, the camera and two instruments are inserted together via a single working incision to perform the surgery. The surgical technique for uniportal VATS is described in detail in our previous publication (10). In whole pleural adhesion, the pulmonary resection usually began after the whole pleural adhesiolysis was completed.

\section{Uniportal VATS adhesiolysis}

The skin incision was usually made in the fifth intercostal space between the mid- and anterior axillary lines, except in right upper lobectomy, where the fourth intercostal space was chosen. The incision length was usually 3 to $3.5 \mathrm{~cm}$. When pleural adhesion was detected, a surgeon's finger was placed into the working incision to create a space by blunt dissection, allowing for insertion of the thoracoscope into the working incision (Figure 1). Meticulous pleural adhesiolysis was then conducted by electrocauterization using a curved instrument (Figure 2). We were able to approach the diaphragm easily when the working incision was made at the fifth intercostal space of the anterior axillary line. From this location, when both the thoracoscope and the surgical instruments are pointed towards the diaphragm, all parts of the diaphragm are accessible with the surgical instruments and adhesiolysis around the diaphragm can be easily performed through the single port. Curved electrocautery was used for adhesiolysis close to the working incision site, followed by adhesiolysis in areas far from the working incision by using an energybased vessel sealing device (harmonic scalpel). In cases where a peripheral tumor was adjacent to the visceral pleura, we always performed adhesiolysis by carrying out extrapleural dissection while the parietal pleura around the tumor was attached to the lung.

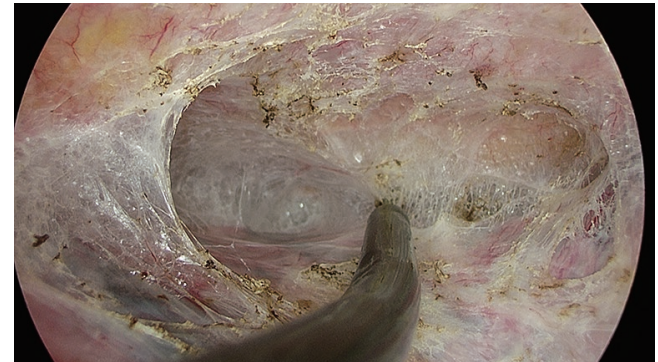

Figure 2 Pleural adhesiolysis is conducted by electrocauterization using a curved instrument.

\section{Multiportal VATS adhesiolysis}

The operation was performed by using a working incision and 3 additional ports. The working incision was made in the fourth intercostal space between the mid- and anterior axillary lines and the 3 additional ports were placed at the seventh intercostal space in the mid-axillary line, the fifth intercostal space under the tip of the scapula, and the sixth intercostal space at the anterior axillary line. Adhesiolysis started with a blunt dissection from the working incision site. The 3 ports were inserted after the space for the port sites was secured, and all ports and the working incision were then used for adhesiolysis.

\section{Statistical analysis}

Clinicopathologic factors were compared using a Student's $t$-test or the Wilcoxon rank sum test for continuous variables and chi-squared or Fisher exact test for categorical variables. The Kaplan-Meier method was used to analyze data collected from the interval between the time of operation and the time of the last follow-up visit and to estimate the recurrence-free survival (RFS) and diseasespecific survival (DSS) rates. Survival of each group was compared by log-rank test. A Cox proportional hazards model was used in a multivariate analysis to identify risk factors for recurrence after surgery. All variables with $\mathrm{P}<0.1$ on univariate analysis were entered into the multivariate analysis. Any $\mathrm{P}<0.05$ was considered statistically significant. Statistical analysis was performed using SPSS version 24.0 software (IBM Corp, Armonk, NY, United States).

\section{Results}

Table 1 shows the comparison of clinicopathologic characteristics between uniportal VATS and multiportal VATS. Sixteen patients underwent uniportal VATS and 
Table 1 Comparison of clinicopathologic characteristics in uniportal VATS and multiportal VATS for patients with stage I and II non-small cell lung cancers with whole pleural adhesion

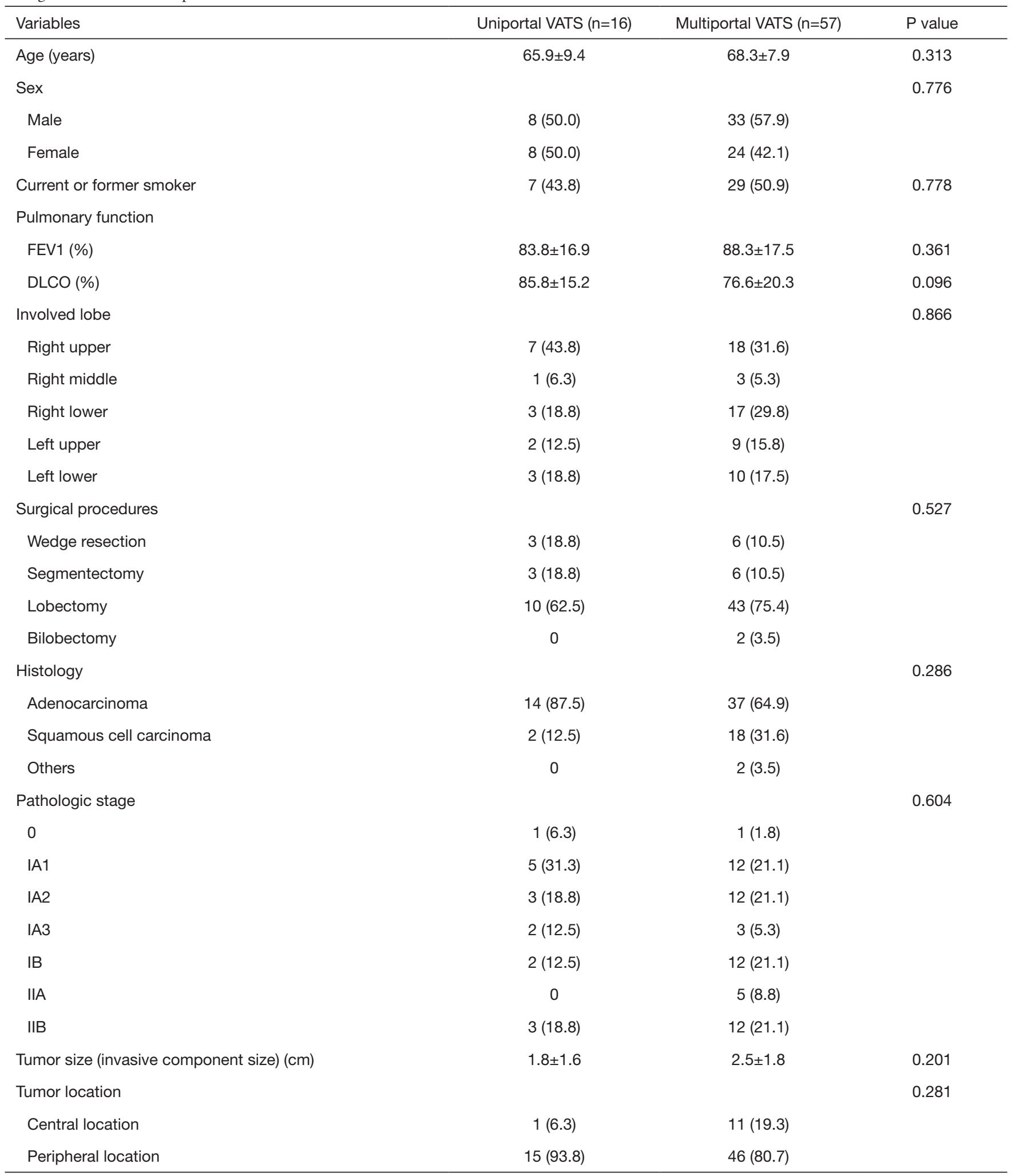

Table 1 (continued) 
Table 1 (continued)

\begin{tabular}{lcc}
\hline Variables & Uniportal VATS $(\mathrm{n}=16)$ & Multiportal VATS $(\mathrm{n}=57)$ \\
\hline Histologic tumor grade & & 0.245 \\
Well differentiated & $8(50.0)$ & $15(26.3)$ \\
Moderately differentiated & $5(31.3)$ & $3(45.6)$ \\
Poorly differentiated & $3(18.8)$ & $16(28.1)$ \\
Pleural invasion & $4(25.0)$ & $13(22.8)$ \\
Visceral pleural invasion & 0 & $4(7.0)$ \\
Parietal pleural invasion & $5(31.3)$ & $30(52.6)$ \\
\hline
\end{tabular}

Data are shown as mean \pm SD or number (percentage). VATS, video-assisted thoracoscopic surgery; SD, standard deviation; FEV1, forced expiratory volume in 1 second; DLCO, diffusing capacity for carbon monoxide.

57 had multiportal VATS. There were no significant differences of clinicopathologic characteristics between the two groups.

\section{Comparison of surgical outcomes between uniportal VATS and multiportal VATS}

Table 2 shows the perioperative and postoperative outcomes for all patients. There were 5 conversions to open thoracotomy in the multiportal VATS group, whereas there were no conversions in the uniportal VATS group. However, the difference of conversion rate was not statistically significant $(\mathrm{P}=0.579)$. There were two conversions from uniportal VATS to multiportal VATS due to endo-stapler direction and adhesiolysis at the diaphragm.

Other perioperative outcomes were also similar between the two groups, as were the postoperative complication rates (31.3\% uniportal VATS vs. 31.6\% multiportal VATS). All complications were resolved during the hospital stay. There was no mortality in all patients.

In subgroup analysis of patients undergoing VATS lobectomy (Table 3), there was no statistical difference in perioperative and postoperative outcomes between uniportal VATS lobectomy and multiportal VATS lobectomy.

\section{Survival analysis of all study patients}

The comparison of RFS between uniportal VATS and multiportal VATS was conducted in all study patients. The median follow-up time for the uniportal VATS patients was 701 days (range, 195-1,169 days) and the median follow- up time for the multiportal VATS group was 1,872 days (range, 151-3,469 days). A total of 16 patients had recurrence, and the sites of recurrence were similar in uniportal VATS and multiportal VATS (Table 4). Since the follow-up time in the uniportal VATS group was short, the 3-year RFS was compared between the two groups. The 3-year RFS after uniportal VATS was $80.0 \%$, and the 3 -year RFS after multiportal VATS was $79.5 \%(\mathrm{P}=0.951)$ (Figure 3).

\section{Survival analysis for stage I non-small cell lung cancer}

RFS was compared for uniportal VATS and multiportal VATS in patients with stage I NSCLC after confirming that there was no statistical difference of clinicopathologic characteristics between the two groups (Table 5).

A total of 6 patients had recurrences after a median followup time of 683 days (range, 195-1,169 days) for the uniportal VATS group and 2,005 days (range, 176-3,366 days) for the multiportal VATS group (Table 6). Three-year RFS after uniportal VATS was $91.7 \%$ and 3-year RFS after multiportal VATS was $89.3 \%(\mathrm{P}=0.999)$ for patients with stage I NSCLC with whole pleural adhesion (Figure 4).

\section{Recurrence risk}

We next analyzed risk factors for recurrence after VATS in patients with stage I NSCLC with whole pleural adhesion (Table 7) by conducting univariate and multivariate analyses using a Cox proportional hazard model to identify the risk factors for recurrence. Significant factors $(\mathrm{P}<0.1)$ on 
Table 2 Comparison of perioperative and postoperative outcomes in uniportal VATS and multiportal VATS

\begin{tabular}{|c|c|c|c|}
\hline Variables & Uniportal VATS ( $\mathrm{n}=16)$ & Multiportal VATS $(\mathrm{n}=57)$ & $P$ value \\
\hline Extent of lymph node dissection & & & 0.764 \\
\hline No mediastinal node dissection & $4(25.0 \%)$ & $13(22.8 \%)$ & \\
\hline Systematic nodal dissection & $6(37.5 \%)$ & $27(47.4 \%)$ & \\
\hline Number of dissected lymph nodes & & & 0.388 \\
\hline Mean \pm SD & $10.0 \pm 9.3$ & $12.6 \pm 11.0$ & \\
\hline Median [Q1-Q3] & 9 [2.3-15.3] & $11[4-19]$ & \\
\hline Anesthetic duration (min) & & & 0.070 \\
\hline Operative time (min) & & & 0.248 \\
\hline Mean \pm SD & $204.0 \pm 70.7$ & $225.9 \pm 65.5$ & \\
\hline Median [Q1-Q3] & $224.5[131.3-260.0]$ & 210 [172.5-268.0] & \\
\hline Intraoperative blood loss (mL) & & & 0.101 \\
\hline Mean \pm SD & $125.0 \pm 414.0$ & $417.0 \pm 432.3$ & \\
\hline Median [Q1-Q3] & $75[50.0-187.5]$ & $300[100-500]$ & \\
\hline Postoperative chest drainage (day) & & & 0.733 \\
\hline Mean \pm SD & $8.1 \pm 7.7$ & $7.2 \pm 8.6$ & \\
\hline Prolonged air leak (>5 days) & 3 & 14 & \\
\hline Chylothorax & 0 & 1 & \\
\hline Atrial fibrillation & 0 & 1 & \\
\hline Pneumonia & 2 & 2 & \\
\hline Bronchial stenosis & 0 & 1 & \\
\hline Operative mortality & 0 & 0 & \\
\hline
\end{tabular}

VATS, video-assisted thoracoscopic surgery; SD, standard deviation.

univariate analysis included age, invasive component size, and lymphovascular invasion. No significant risk factors for recurrence emerged when these variables were entered into the multivariate model. Uniportal VATS was not a significant risk factor for recurrence in this analysis.

\section{Discussion}

VATS is considered a challenging procedure when there is severe whole pleural adhesion during pulmonary resection for lung cancer (11). Pleural adhesiolysis through VATS 
Table 3 Comparison of perioperative and postoperative outcomes in uniportal VATS lobectomy and multiportal VATS lobectomy

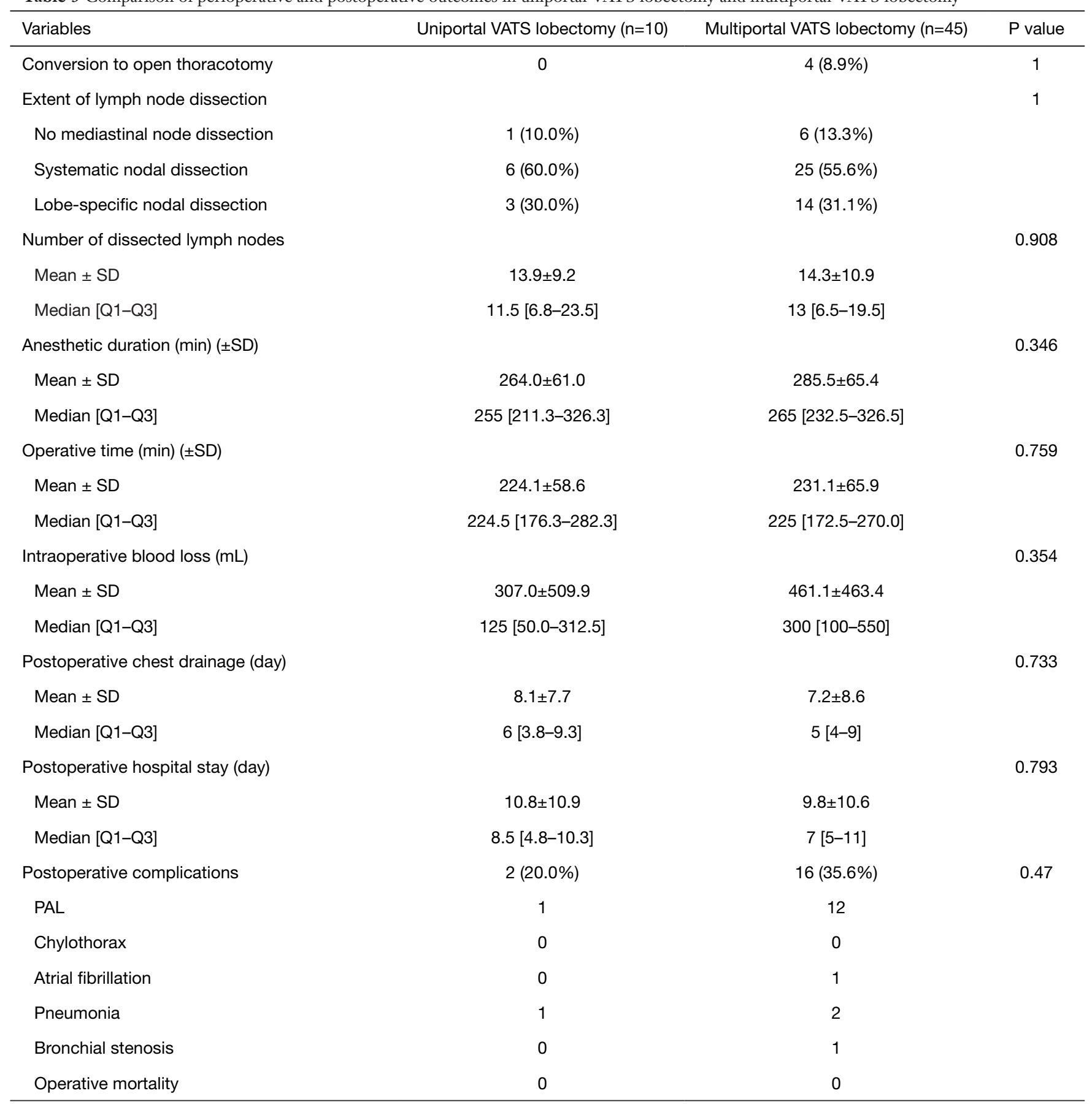

VATS, video-assisted thoracoscopic surgery; SD, standard deviation; Q1-Q3, quartile 1 - quartile 3; PAL, prolonged air leak (>5 days).

became possible with advances in surgical instruments and surgical skills, and lung cancer surgery with whole pleural adhesion can now be performed with multiportal VATS (12). However, until we completed the uniportal VATS training program at the Shanghai Pulmonary Hospital, we thought that lung cancer surgery with uniportal VATS would be impossible in patients with whole pleural adhesion. Since completing the training program, we have realized that all lung cancer surgery can be performed with uniportal VATS (10). In fact, when adhesiolysis is performed with 
Table 4 Summary of recurrence in all patients

\begin{tabular}{lcc}
\hline Variables & Uniportal VATS $(\mathrm{n}=16)$ & Multiportal VATS $(\mathrm{n}=57)$ \\
\hline Sites of recurrence & & 0.771 \\
Locoregional recurrence & $1(6.3 \%)$ & $6(10.5 \%)$ \\
Distant recurrence & $2(12.5 \%)$ & $4(7.0 \%)$ \\
Both & 0 & $3(5.3 \%)$ \\
\hline
\end{tabular}

Locoregional, recurrence within ipsilateral hemithorax including pleura and mediastinal lymph nodes; Both, locoregional recurrence + distant recurrence.

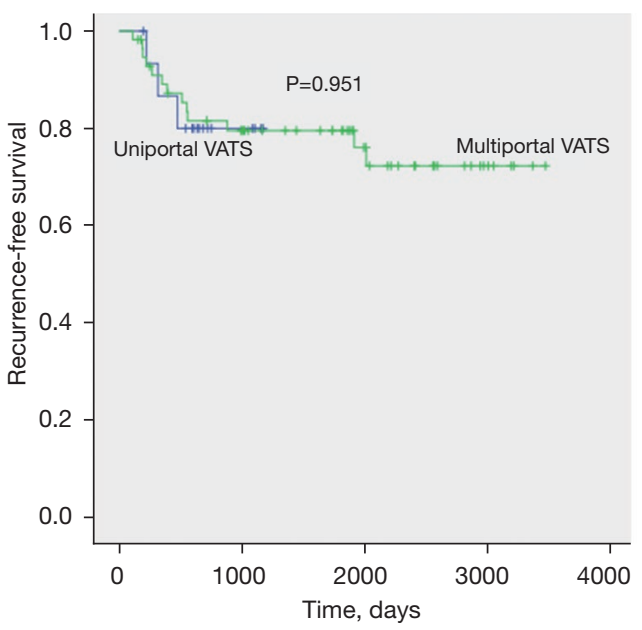

Figure 3 Three-year recurrence-free survival (RFS) is $80.0 \%$ after uniportal video-assisted thoracoscopic surgery (VATS) and $79.5 \%$ after multiportal VATS in patients with stage I and II non-small cell lung cancer with whole pleural adhesion $(\mathrm{P}=0.951)$.

uniportal VATS, the field of view is better than with multiportal VATS, and the adhesion can be lysed in all directions using a curved instrument. The surgical instruments and techniques in uniportal VATS are continuously evolving, and the potential for uniportal VATS in lung cancer surgery now seems to be without further limitation $(1,3,7,13)$.

In this study, the surgical outcome of uniportal VATS was not inferior to that of multiportal VATS. The operative time was similar between uniportal VATS and multiportal VATS. The thoracotomy conversion rates were not statistically different between the two groups. Conversion to thoracotomy occurred in 5 patients who underwent multiportal VATS (4 due to severe pleural adhesion, and 1 due to calcified perivascular lymph nodes), while there were no conversions to thoracotomy in the patients who underwent uniportal VATS. There were two conversions from uniportal VATS to multiportal VATS. In the first case, all adhesiolysis was completed with uniportal VATS, but when dividing the pulmonary artery, the direction of the endostapler was unsatisfactory, and one additional thoracoscopic port was created. In the end, we did not use the newly created thoracoscopic port, as we were eventually able to divide the pulmonary artery through the working port. In the second case, a thoracoscopic port was added to help detach the adhesions between the diaphragm and lungs, but the contribution of the additional port to adhesiolysis was minimal. Both of these cases were in the early stages of initiating uniportal VATS, and since the additional port was not used for the main surgery, we kept both cases in the uniportal VATS group.

In our previous study, pleural adhesion was not a risk factor for recurrence when lung cancer was treated by multiportal VATS (12). To date, there have been few studies to evaluate the effect of uniportal VATS on the prognosis of NSCLC with whole pleural adhesion. In this study, the uniportal and multiportal VATS groups had similar 3-year RFS and DSS rates, and uniportal VATS was not a risk factor for recurrence in patients with stage I NSCLC with whole pleural adhesion. Therefore, we have concluded that uniportal VATS need no longer be limited in lung cancer surgery with whole pleural adhesion. Of course, it is difficult to generalize the results of this study because the number of cases in this study is small and the follow-up period is short. This study is an initial study to evaluate whether it is appropriate to perform uniportal VATS regardless of the presence or absence of pleural adhesion even as the efficacy of uniportal surgery in treating lung cancer with whole pleural adhesion is not well known. We now believe that there is no difference in difficulty between uniportal VATS and multiportal VATS when performing whole pleural adhesiolysis. Therefore, if more cases are gathered in the future, more accurate results will be obtained.

Pleural adhesions are usually caused by past inflammation 
Table 5 Comparison of clinicopathologic characteristics in patients undergoing uniportal VATS and multiportal VATS for stage I non-small cell lung cancer with whole pleural adhesion

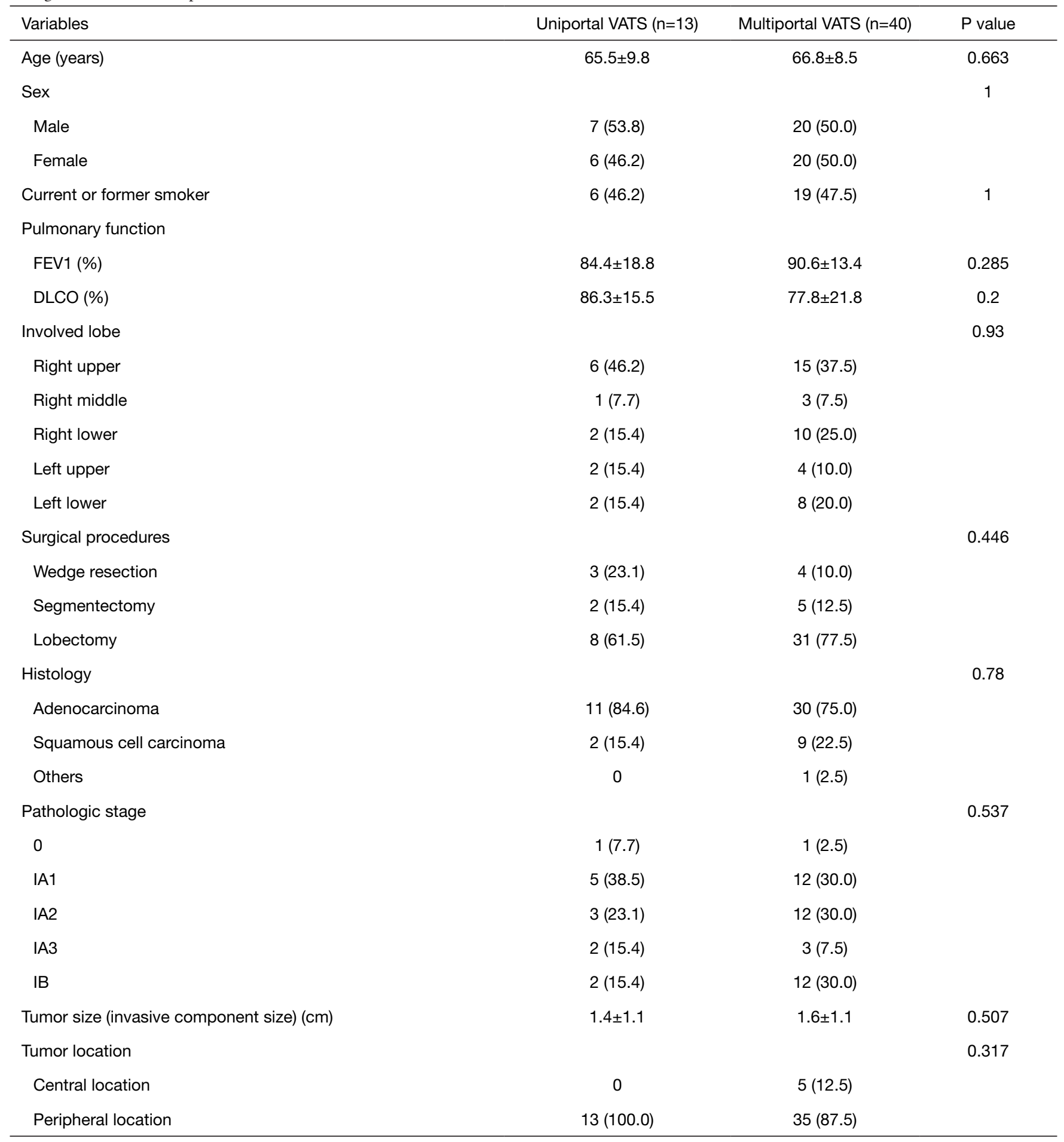

Table 5 (continued) 
Table 5 (continued)

\begin{tabular}{lcc}
\hline Variables & Uniportal VATS $(\mathrm{n}=13)$ & Multiportal VATS $(\mathrm{n}=40)$ \\
\hline Histologic tumor grade & & 0.651 \\
Well differentiated & $7(53.8)$ & $15(37.5)$ \\
Moderately differentiated & $4(30.8)$ & $16(40.0)$ \\
Poorly differentiated & $2(15.4)$ & $9(22.5)$ \\
Visceral pleural invasion & $2(15.4)$ & $9(22.5)$ \\
Lymphovascular invasion & $3(23.1)$ & $15(37.5)$ \\
\hline
\end{tabular}

Data are shown as mean \pm SD or number (percentage). VATS, video-assisted thoracoscopic surgery; SD, standard deviation; FEV1, forced expiratory volume in 1 second; DLCO, diffusing capacity for carbon monoxide.

Table 6 Summary of recurrence in patients with stage I non-small cell lung cancer with whole pleural adhesion

\begin{tabular}{lcc}
\hline Variables & Uniportal VATS $(\mathrm{n}=13)$ & Multiportal VATS $(\mathrm{n}=40)$ \\
\hline Sites of recurrence & $1(7.7 \%)$ & $3(7.5 \%)$ \\
Locoregional recurrence & & 1.000 \\
Distant recurrence & & 0 \\
Both & 0 & $2(5.0 \%)$ \\
\hline
\end{tabular}

Locoregional, recurrence within ipsilateral hemithorax including pleura and mediastinal lymph nodes; Both, locoregional recurrence + distant recurrence.

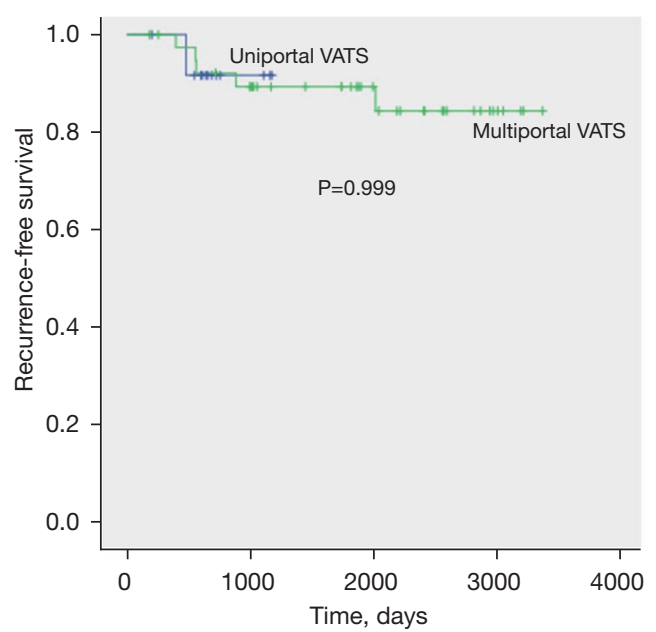

Figure 4 Three-year recurrence-free survival (RFS) is $91.7 \%$ after uniportal video-assisted thoracoscopic surgery (VATS) and $89.3 \%$ after multiportal VATS in patients with stage I non-small cell lung cancer with whole pleural adhesion $(\mathrm{P}=0.999)$.

of the pleura, and the degree of pleural adhesion varies from patient to patient. Some pleural adhesions can be detached easily by blunt dissection, and some can be detached only by sharp dissection with electrocauterization. The degree of difficulty of surgery will inevitably vary for each patient with pleural adhesion. However, there is no uniform measure of the degree of pleural adhesion. In this study, for a more precise analysis, all patients with partial pleural adhesion were excluded and only patients with whole pleural adhesion were included. Patients with whole pleural adhesion posed similar difficulty in surgery because the pleura was not well separated by blunt dissection. Therefore, unlike other studies related to pleural adhesion (14), this study compared uniportal VATS and multi-portal VATS only for patients with whole pleural adhesion.

This study had several limitations that should be considered. Firstly, we used a retrospective study design. Secondly, we obtained data from a single institution with a small sample size, so generalizing our results is difficult. However, there are advantages to the use of data of patients undergoing surgery in one hospital. It may be more accurate to compare uniportal VATS and multiportal VATS because the treatment protocols are standardized and we are comparing surgical techniques with the same surgeons. If more cases are gathered in the future, more robust results will be obtained. Finally, it has only been a 
Table 7 Univariate analysis and multivariate analysis of risk factors for recurrence after uniportal VATS in patients with stage I non-small cell lung cancer with whole pleural adhesion

\begin{tabular}{|c|c|c|c|}
\hline Variables & HR & $95 \% \mathrm{Cl}$ & $P$ value \\
\hline Age & 1.141 & $1.019-1.277$ & 0.022 \\
\hline Sex (male) & 0.907 & $0.183-4.498$ & 0.905 \\
\hline Smoker & 0.574 & $0.105-3.136$ & 0.522 \\
\hline DLCO (\%) & 1.002 & $0.961-1.045$ & 0.912 \\
\hline Lobe & & & 0.731 \\
\hline Right upper (reference) & 1 & & \\
\hline Right middle & 0 & 0 & 0.991 \\
\hline Left lower & 5.152 & $0.466-56.976$ & 0.181 \\
\hline Sublobar resection & 0.669 & $0.077-5.803$ & 0.715 \\
\hline Uniportal VATS & 1.002 & $0.109-9.190$ & 0.999 \\
\hline Extent of Lymph node dissection & & & 0.948 \\
\hline No mediastinal node dissection (reference) & 1 & & 0.745 \\
\hline Systematic nodal dissection & 1.457 & $0.151-14.101$ & 0.797 \\
\hline Lobe-specific nodal dissection & 1.370 & $0.124-15.122$ & \\
\hline Histology & & & 0.105 \\
\hline Well differentiated (reference) & 1 & & \\
\hline Moderately differentiated & 6.758 & $0.785-58.195$ & 0.082 \\
\hline Poorly differentiated & 0 & 0 & 0.982 \\
\hline Number of dissected lymph nodes & 1.029 & $0.949-1.115$ & 0.491 \\
\hline Visceral pleural invasion & 2.890 & $0.337-24.809$ & 0.333 \\
\hline Lymphovascular invasion & 5.565 & $1.002-30.914$ & 0.050 \\
\hline \multicolumn{4}{|l|}{ Multivariate analysis } \\
\hline Age & 1.092 & $0.974-1.225$ & 0.131 \\
\hline Invasive component size & 1.662 & $0.623-4.437$ & 0.310 \\
\hline Lymphovascular invasion & 2.553 & $0.354-18.426$ & 0.353 \\
\hline
\end{tabular}

VATS, video-assisted thoracoscopic surgery; HR, hazard ratio; Cl, confidence interval; FEV1, forced expiratory volume in 1 second; DLCO, diffusing capacity for carbon monoxide. 
few years since uniportal VATS was initiated, and longterm survival rates have not been compared. However, most cases of NSCLC are known to recur within two years after surgery (15), and early recurrence has proven to be an accurate reflection of long-term outcomes (16). In addition, this study is more focused on the feasibility of uniportal VATS as a surgical technique in cases of lung cancer with whole pleural adhesion than on comparing the prognosis between uniportal VATS and multiportal VATS. More detailed results can be derived in the future if more and longer follow-up data are accumulated.

In conclusion, uniportal VATS may be a feasible surgical approach for the treatment of lung cancer with whole pleural adhesion. Uniportal VATS was not inferior to multiportal VATS in surgical outcome or shortterm prognosis in patients with whole pleural adhesion. Additional research in a larger pool of data may more accurately depict patient outcomes.

\section{Acknowledgments}

We thank the native English-speaking professional (BioMed Proofreading, LLC) for refining the written content.

Funding: None.

\section{Footnote}

Reporting Checklist: The authors have completed the STROBE reporting checklist. Available at https://tcr. amegroups.com/article/view/10.21037/tcr-21-2113/rc

Data Sharing Statement: Available at https://tcr.amegroups. com/article/view/10.21037/tcr-21-2113/dss

Conflicts of Interest: All authors have completed the ICMJE uniform disclosure form (available at https://tcr.amegroups. com/article/view/10.21037/tcr-21-2113/coif). The authors have no conflicts of interest to declare.

Ethical Statement: The authors are accountable for all aspects of the work in ensuring that questions related to the accuracy or integrity of any part of the work are appropriately investigated and resolved. The study was conducted in accordance with the Declaration of Helsinki (as revised in 2013). This study was approved by the institutional review board of Eunpyeong St. Mary's Hospital at the Catholic University of Korea (Referral number:
PC21RISI0115) and individual consent was waived.

Open Access Statement: This is an Open Access article distributed in accordance with the Creative Commons Attribution-NonCommercial-NoDerivs 4.0 International License (CC BY-NC-ND 4.0), which permits the noncommercial replication and distribution of the article with the strict proviso that no changes or edits are made and the original work is properly cited (including links to both the formal publication through the relevant DOI and the license). See: https://creativecommons.org/licenses/by-nc-nd/4.0/.

\section{References}

1. Sihoe ADL. Uniportal Lung Cancer Surgery: State of the Evidence. Ann Thorac Surg 2019;107:962-72.

2. Gonzalez D, Paradela M, Garcia J, et al. Single-port videoassisted thoracoscopic lobectomy. Interact Cardiovasc Thorac Surg 2011;12:514-5.

3. Gonzalez-Rivas D, Fieira E, Delgado M, et al. Uniportal video-assisted thoracoscopic lobectomy. J Thorac Dis 2013;5 Suppl 3:S234-45.

4. Bulgarelli Maqueda L, García-Pérez A, Minasyan A, et al. Uniportal VATS for non-small cell lung cancer. Gen Thorac Cardiovasc Surg 2020;68:707-15.

5. Bertolaccini L, Batirel H, Brunelli A, et al. Uniportal video-assisted thoracic surgery lobectomy: a consensus report from the Uniportal VATS Interest Group (UVIG) of the European Society of Thoracic Surgeons (ESTS). Eur J Cardiothorac Surg 2019;56:224-229. Erratum in: Eur J Cardiothorac Surg 2019;56:628-9.

6. Hernandez-Arenas LA, Purmessur RD, Gonzalez-Rivas D. Uniportal video-assisted thoracoscopic segmentectomy. J Thorac Dis 2018;10:S1205-14.

7. Gonzalez-Rivas D, Sihoe ADL. Important Technical Details During Uniportal Video-Assisted Thoracoscopic Major Resections. Thorac Surg Clin 2017;27:357-72.

8. Gonzalez-Rivas D, Yang Y, Ng C. Advances in Uniportal Video-Assisted Thoracoscopic Surgery: Pushing the Envelope. Thorac Surg Clin 2016;26:187-201.

9. Sihoe ADL, Gonzalez-Rivas D, Yang TY, et al. Highvolume intensive training course: a new paradigm for video-assisted thoracoscopic surgery education. Interact Cardiovasc Thorac Surg 2018;27:365-71.

10. Moon Y. Initial experience with uniportal video-assisted thoracoscopic surgery for the treatment of lung cancer performed by a surgeon who did not have previous 
experience performing multiportal thoracoscopic surgery: a single center retrospective study. J Thorac Dis 2020;12:1972-81.

11. Li SJ, Zhou K, Wu YM, et al. Presence of pleural adhesions can predict conversion to thoracotomy and postoperative surgical complications in patients undergoing video-assisted thoracoscopic lung cancer lobectomy. J Thorac Dis 2018;10:416-31.

12. Moon Y, Choi SY, Moon MH. The prognosis of stage I non-small cell lung cancer with visceral pleural invasion and whole pleural adhesion after video-assisted thoracoscopic lobectomy: A single center retrospective study. J Thorac Dis 2020;12:5729-38.

13. Sihoe AD. The evolution of minimally invasive thoracic surgery: implications for the practice of uniportal thoracoscopic surgery. J Thorac Dis 2014;6:S604-17.

14. Nakada T, Noda Y, Kato D, et al. Risk factors and cancer recurrence associated with postoperative complications after thoracoscopic lobectomy for clinical stage I nonsmall cell lung cancer. Thorac Cancer 2019;10:1945-52.

15. Tremblay L, Deslauriers J. What is the most practical, optimal, and cost effective method for performing followup after lung cancer surgery, and by whom should it be done? Thorac Surg Clin 2013;23:429-36.

16. Kiankhooy A, Taylor MD, LaPar DJ, et al. Predictors of early recurrence for node-negative $\mathrm{t} 1$ to $\mathrm{t} 2 \mathrm{~b}$ non-small cell lung cancer. Ann Thorac Surg 2014;98:1175-83.

Cite this article as: Choi SY, Moon Y. Uniportal video-assisted thoracoscopic surgery (VATS) for the treatment of early-stage lung cancer with whole pleural adhesion. Transl Cancer Res 2022;11(1):72-84. doi: 10.21037/tcr-21-2113 